\title{
ON THE TREATMENT
}

\section{or \\ S I N U O US ULCERS.}

By HENRY DEWAR, M.D. F.R.S. Ed.

FELLOW OF THE ROYAL COLLEGE OF PHYSTCLANS OF EDINBURGH, AND LECTURER ON THE THEORY AND PRACTICE

OF MEDICINE.

COMMUNICATED BY

D R. R O G E T.

Read June 25, 1816.

"What the knife cures, it partly destroys; what the bandage cures, it saves."

John Bell's Principles of Surgery:

FINDING the modern treatment of suppurating sinuses to labour under great deficiencies, I took occasion last summer to circulate some observations on the subject in the military hospitals of Flanders, after the battle of Waterloo, in the form of a letter to a friend employed in that service. They first occurred to me in the year 1799 , when I served as hospital mate at Colchester on the return of the British troops from the Helder, although I was not then sufficiently sanguine to make any attempt to call the attention of the profession to 
the subject. I hope that the following observations, if allowed a place in the Medico-Chirurgical Transactions, will attract the general attention of professional men, and will secure for the subject the notice which is due to it in future systematic books of surgery.

The importance of sinuous ulcers, their frequent tediousness, and the want of success experienced in this department of practice, have induced authors to give a variety of directions as to their treatment. At one time large poultices are recommended for promoting suppuration; at another, acrid injections or the introduction of setons, for exciting a gentle inflammation favourable to adhesion. We are generally advised to lay open a sinus along its whole course, for the complete discharge of the matter, or to make for that purpose a new artificial orifice, called a counter-opening. Most writers say nothing of the effects of compression, and none describe the mode of applying it with that fulness and particularity which are necessary. Hence sinuses, which might be speedily healed up by good management, are suffered to spread without resistance, and to destroy the patient by profuse suppuration. In other instances extensive incisions are made, which give unnecessary pain, and retard the cure by the extent of artificial injury which they occasion; and, even where compresses 
and bandages are used, they are not managed in such a manner as to secure the adhesion of the sides of the sinus.

It is to the neglected subject of the order and gradation of the pressure applied, that I now wish to call the attention of the Society. I shall therefore describe it as adapted to those cases in which it is attended with most conspicuous benefit; namely, extensive sinuses among the muscles of the thigh. These occur chiefly in consequence of abscesses formed under the fascia lata, and of gunshot, and other deep-seated wounds. The anatomy of the thigh subjects it in a particular manner to extensive sinuses. The tough unyielding substance of the fascia prevents the matter from pointing outwards; so that it extends interiorly in all directions. This extension is promoted by the looseness of the cellular membrane, the number and length of the muscles that pass along the femur, their narrow and distant attachments, together with the divergency of some of them at their upper ends, where they are connected with the bones of the pelvis. In consequence of these circumstances which deprive the cellular membrane of firm support, the pus quickly diffuses itself through it, and from its presence as a foreign body, and probably from a specific action on the living parts, produces such a spreading of the suppurating process, that the quantity of matter secreted soon becomes enormous. Large sinuses thus formed after gun-shot 
wounds, have been too generally considered in the army as bad cases, in which extensive and continued suppuration is unavoidable, and the helpless practitioner is obliged to wait the uncertain issue of a tedious process. Where the whole muscles of the thigh are separated like those of a dissected limb, the extent of the evil, and the large bloodvessels that are in the way, render it impossible to lay open all the branches of the sinus, and the formidable nature of the disease seems, in fatal cases, to exonerate the practitioner from all blame. The methods of dressing in general use may be reduced to two; one is, to put a loose roller over the whole, or to be satisfied with a covering in the form of a towel, containing a large poultice; that the parts may be kept easy, and that the matter may have a free exit. Under this management there may be an overflow, but there is no evacuation. The cavity continues so much filled with matter, that one laborious and altogether ineffectual part of daily duty is, to force it out by pressing the integuments from the extremities towards the orifice of the cavity. The disease is still allowed to extend interiorly, and no decisive effort is made to bring on a process of adhesion. The other mode of bandaging is, to apply a roller with considerable tightness, but with equal pressure over the whole thigh, beginning at the knee-joint, and carrying it regularly upwards by successive turns, passing it over the orifice in its way, and fixing it at the upper part of the thigh. This application is equally ineffectual 
with the preceding. It cannot produce an immediate adhesion of parts so extensively divided, and kept asunder by a profusely secreted pus; and it leaves them in the same state by pressing those parts which are nearest to the orifice, as much or more than those which are remote. In general, it so presses the orifice itself, as to retain forcibly the whole pus formed between the times of dressing, and thus to increase the irritation excited by the presence of a foreign body. It even impels the matter beyond the parts to which any portion of the bandage is applied, and thus greatly promotes the extension of the sinus.

A case of this kind, which I was employed to dress in the routine of duty, had been bandaged in the manner now described. The process of suppuration was advancing, there was not the slightest tendency to a recovery, and the strength of the patient gradually declined. The consequences of the treatment which I am now to recommend fulfilled my best hopes, and furnished a useful lesson for the practical surgeon. Though no journal of the case was kept, the facts will be easily understood, and the utility of the practice, I hope, established without that aid; as its merits do not depend entirely on the results of clinical experiments, but are plausibly supported by the soundest data. It has long, indeed, escaped general attention, and therefore requires minuteness of detail. But it is worthy of all the pains that can be taken 
to inculcate it, and if the object can be thus obtained, there will be little reason to repine at any charges of superfluous importunity which a reiteration of zealous expressions may incur.

The following is the method to be substituted for the preceding expedients. A few turns of the roller should first be made with considerable pressure over one extremity of the femur, and then over the other, so as to reach with all possible certainty the extremities of the large sinus, into which the whole cellular interstices of the parts have been converted. It is safer to begin beyond the sinus than to run any risk of falling short of its extremities; and, in some cases, it might be proper to increase our security by means of partial compresses extending somewhat higher than it is possible to apply the turns of the roller itself. It is now fixed in its situation with a pin. A considerable pressure is easily borne, as no high inflammation is present, and the evacuation of the pus, by reducing the circumference of the limb, soon relieves the veins from any turgescence arising from the pressure to which they may have been at first subjected. In country practice, when a surgeon has been newly called to an old case of this kind, and a considerable interval may elapse before he is to repeat his visit, the swelling of the lower part of the limb may be obviated by bandaging it upwards from the toes. After fixing the bandage on the thigh at the degree of pressure which $I$ have 
described, the surgeon may, if he chooses, make two or three lighter turns on the tumid part to assist the depletion of it; taking care that these press so lightly, as in no degree to counteract the operation of the first turns made at the extremities of the sinus. The change which this application produces is almost immediate. Part of the matter with which the integuments had been distended, is irresistibly forced a certain way towards the orifice; and no newly secreted matter is suffered to lodge in that quarter. On the second day, the limb is found somewhat reduced in size, and the bandage may now be applied more extensively. On the third day, it may be so applied as to be kept on for several days without alteration. The same degree of pressure is always to be continued over the extremities of the sinus, and several additional turns are to be made, gradually looser, alternately above and below the orifice, and approaching to it in both directions, but not reaching it. If there are two orifices, one of them, by which the matter can be freely brought away, is to be left uncovered with the bandage, and the other allowed to heal up. There is no necessity for selecting the most de. pendent one for that purpose, as any advantage derived from the tendency given to the course of the matter by its own weight, is not worthy of attention under a treatment implying means of evacuation otherwise so powerful. The anterior oriflee will often be found the most eligible, as it is examined and dressed with greatest convenience. 
During the alternate application of the bandage to the higher and the lower part of the thigh, it is frequently and variously crossed on the side of the limb opposite to the open orifice, and thus a propulsion of the pus is commanded in every direction to that outlet. A considerable part of the surface surrounding it is left uncovered, and the bandage is finally fixed. Over the orifice such light dressings are subsequently applied as will make no resistance to the discharge of the purulent matter. The firm propelling bandage is kept on without alteration, except when it becomes loose in consequence of a reduction in the size of the limb; although cleanliness requires the dressing immediately over the orifice to be changed daily or oftener. Thus all unnecessary trouble is prevented, an object which is sometimes of importance in securing the more perfect performance of those offices which are really necessary.

When no further cause of disease has existed besides those now mentioned, and when the pus is the only foreign body contained in the cavity, the process of adhesion soon begins at the extremities of the sinus. The principles of adhesion have been very well understood since Mr. John Hunter wrote on the subject; but it will be useful on the present occasion to remark, that a considerable pressure over relaxed parts promotes it independently of the evacuation of the pus, by affording mechanical. support, in the same manner as it promotes the 
healing of the relaxed surfaces of ulcers. It thus supersedes the stimulant injections which have been so often employed for the purpose of inducing rawness. The process of adhesion advances by successive steps from the extremities towards the orifice of the sinus, which even in the end requires but little pressure. The discharge quickly diminishes, and shews a proportional diminution of internal disease, and the parts heal in the same kindly manner as a superficial abscess among firm integuments after the pus has been discharged. In the case to which I have alluded, these effects were amply and speedily obtained. Both the patient and the medical gentleman who saw it were surprised at the celerity of the cure. But it was not to my knowledge made the subject of any discussion or conversation, farther than by being mentioned as a remarkably fortutate case. The only circumstances, then, systematically attended to in the application of bandages, were the neatness and smoothness of their appearance, and the utility of the uniform pressure recommended by Mr. Bayn. ton in superficial ulcers. A pressure greatest at the remote part, and gradually declining towards the outlet of a sinus, to give the compressed fluid a safe direction, and to secure the commencement of adhesion in that quarter in which it is not liable to future interruption, though obvious dictates of surgical science, were never thought of.

As all the books now put into the hands of young 
surgeons are deficient in this particular, I have made an extensive inquiry into the works of the older writers, to learn if a method so obvious had not formerly occurred. I have found the practice in this respect somewhat varied, but in general negligent. Avicenna directs the surgeon to begin his compression at the orifice of a sinus. Others more properly prescribe an order the reverse of this, yet leave the rules of management in other respects vague. Hence no succession is understood to" be necessary in the degree of force; the pressure is in almost all cases too slight, and applied over too narrow an extent of surface. It ought always to be applied over the whole thigh in the manner which has been here described, even though the limits of the sinus should be inconsiderable. On reading the scanty observations to be met with in books on the mechanical part of the treatment of sinuses, a young surgeon will at most figure to his mind a narrow passage, the sides of which he must endeavour to bring together. Even if he justly thinks of applying pressure first over the fundus of it alone, which is rarely the case, his views extend no farther than to the compression of the part itself, and the instrument which he contemplates for the fulfilment of his object is a compress nearly of the shape and size of the sinus. On actual application, this may be suspected of being liable to shift, and the sinus may then extend in directions in which nothing was apprehended. There may be narrow communications with the adjacent parts 
of the cellular membrane; and the effect of a limited pressure, though directed accurately over the present sinus, will be, to send part of the matter, in different lateral or ulterior directions, extending the bounds of some unknown branch of the sinus, or creating a new one in the looser cellular membrane. No directions are given to keep this pressure in a fixed state; it is liable therefore to be altered; the internal surfaces are shifted at every time that the dressings of the orifice are renewed; and the process of adhesion is perpetually disturbed. An extended compression of the neighbouring parts is necessary to secure the expulsion of the matter; steadiness is necessary to the advancement of adhesion. The general method here pointed out is on various accounts required to retain the parts in such a disposition as will obviate the extension and terminate the duration of a state of disease.

I have had the pleasure of finding that all the older writers are not equally deficient in their practical precepts on this subject. Galen, Etius, Paulus Fgineta, Heister and Tagault, give luminous and precise directions on the gradation of pressure. Those of Galen are to be found in his treatise entitled $\mathrm{T} \alpha$ ' is not necessary to transcribe the whole passage, the style of which is somewhat prolix; but those who take an interest in the antiquities of medical literature will find pleasure in reading it. I shall 
only select from it the following expressions. $A$ i

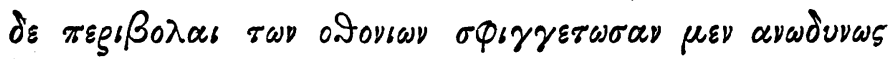

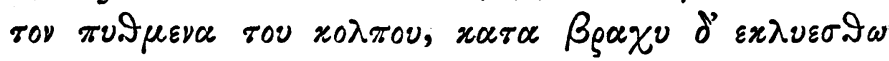

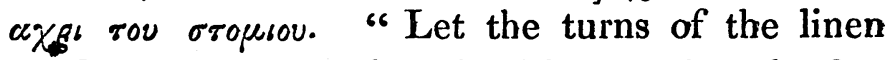
bandages constrict (though without pain) the further end of the sinus, and be made gradually looser towards the orifice." After some more minute

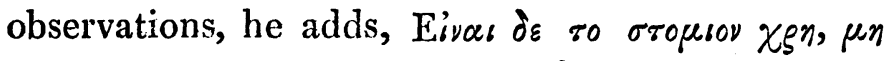

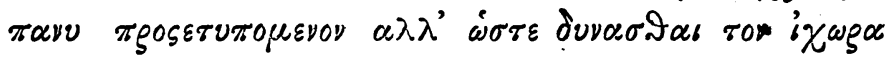

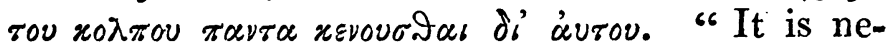
cessary that the orifice should not be at all compressed, but kept so free that the whole matter of the sinus may be evacuated by it." REtius and Paulus deliver the same precepts and nearly in the words of Galen. The directions of Heister to the same purpose, are contained in Part I. Book 2. Chap. II. \2. of his Institutiones Chirurgica. Yet this author appears not to entertain a high enough idea of the degree of pressure necessary to secure the object, as he considers the dependent situation of the orifice as a matter of considerable importance. In other respects, his directions so completely coincide with those of Galen, that it is quite unnecessary to quote the passage. The case is the same with Tagault in Book 3. Ch. 17. of his Treatise on Surgery. Fabricius and many other writers entertained no expectation of the possibility of producing universal and permanent adhesion in large sinuses by compression. This writer says that, in obstinate cases, he sometimes closed and healed up the orifice and the adjoining parts, to gratify the 
patient, though fully aware that a fresh abscess would in a short time be formed. The remedies in which he and his cotemporaries chiefly confided were the agglutinantia. It is not out of place here to remark, to the honour of Galen's discernment, that though he uses an equivalent term, $s \alpha x_{0} \lambda \lambda \eta$ rovra, to denote such medicines as produce adhesion, he does not include in that list, some which came into common use in the subsequent dark ages of surgery, such as isinglass and others, which were expected to operate on the same principle with carpenter's glue; but warns the practitioner against them, as substances which can only have the same effect with the lodging of foreign and impure matter. His $\varkappa_{0} \lambda \lambda \eta \sigma o v \tau \alpha$ are wine and other stimulants, which might at the present moment be used on the most approved and accurate principles of surgery.

The mode of bandaging now described will possess considerable advantages in cases of more complicated disease. If pieces of cloth, splinters of bone, or other foreign bodies are present, it will tend to expel them by the ready evacuation which it effects. If it does not expel them, yet, by reducing the local disease to the spot in which they are lodged, it procures for the practitioner sure information of their presence, and thus gives him an opportunity of using his best endeavours to remove them. In all cases it must facilitate recovery by circumscribing the local disease; even where 
the bone is fractured by a musket shot, it will have the effect of reducing the disease more nearly to that of a common compound, and soon to that of a simple fracture.

When the discharge from a sinuous ulcer is fetid and ichorous, shewing an unhealthy state of the parts, some might be disposed to expect less advantage from this mode of bandaging. This most frequently occurs among numerous tendons or ligaments, as in the ham or in the foot, and arises in some measure from the nature of that species of structure, and the forcible separation of the parts attending it; and the disease will in some degree yield to the powerful compression of a bandage diminishing the contrast between the firmness of the ligaments and the openness of the intervening spaces. In many cases a healthy action may thus be established, especially if the aid of stimulant injections is superadded.

It is scarcely necessary to describe the modes in which the same principles may be applied to the treatment of sinuses in other parts of the body. In the arm and leg there will be no occasion to deviate from the exact form of the bandage as already described. When a sinus exists under the integuments of the foot extending among the ligaments and tendons, maný respectable surgeons lay them open the whole length of the foot, and then 
use means for healing up the cavity by granulation. This harsh operation may evidently be superseded by the bandage applied according to the principles above explained, and with a tightness proportioned to the resistance which it receives from the structure of the parts.

In sinuses occurring among the integuments of the trunk, greater address is required, and in many cases the success of the most judicious management must be uncertain, as we have not such points of support as will afford complete security against the extension of the sinus to the great cavities of the body. In some cases a compress in the form of a crescent might prove useful for preventing the spreading of the suppuration in various directions, and thus promoting the tendency of it to the orifice. The open part between the horns of the crescent will correspond to the outlet.

In situations in which the inequalities of the bones are unfavourable to the fairness of the pressure, compresses may be interposed suited to the shape of the interstices. Le Dran describes a very instructive case of suppuration under the pectoral muscle, which deserves to be mentioned as affording a beautiful illustration of this method of cure, under slight variation of the instruments employed, as suited to the situation of the disease. An abscess had begun in the axilla of his patient. 
and extended under the pectoral muscle to the clavicle. This gentleman made an opening at the lower part of that muscle, and ascertained by the probe that the sinus extended to the periosteum of the clavicle. He dressed it for several days with digestive ointment, and introduced a dossil of a flat shape armed with this ointment to the extremity of the sinus. In eight days after, he dressed it with what he calls a mundificant, and then (to use his own language) left the bottom of it to nature. These means may have been very useful in this case to produce adhesion in a part to which compression could not be applied with full security of success. But his practice in that respect seems to have been partly founded on a persuasion of the necessity of producing such a secretion as would not create disturbance by its unhealthy qualities. But it was now, when he considered himself as leaving the case to nature, that he shewed most clearly the soundness of his judgment. To assist nature, he made use of an expulsive compress, which was long and narrow. This was first applied transversely under the clavicle, one close to the bone. Next day, another was applied of the same length, but somewhat broader, and the breadth of these compresses was daily increased, a free drain being always left for the matter: The quantity of pus was very extraordinary; but the sinus was healed on the thirteenth day, and the wound soon cicatrized by being methodically 
dressed. He warns the surgeon not to apply the expulsive compress to the whole extent of the sinus, because it may compress too strongly between the remote part of the cavity and the orifice, and thus counteract the re-union by restraining the evaeuation of the pus. He might have added that it is more difficult, even though the matter is evacuated, to produce a healthy adhesion simultaneously over a large extent of diseased parts than to effect the adhesion by successive steps.

Edinburgh, 29 June, 1816. 